\title{
Range of neuropsychiatric disturbances in patients with Parkinson's disease
} Dag Aarsland, Jan P Larsen, Neh Geok Lim, Carmen Janvin, Karen Karlsen,
Elise Tandberg, Jeffrey L Cummings most previous studies on psychopathology in Parkinson's disease have focused on one or few psychiatric diagnoses, such as depression, ${ }^{1}$ psychosis, ${ }^{2}$ and cognitive impairment. ${ }^{3}$ Little is known about the range of neuropsychiatric syndromes occurring in patients assessed simultaneously for a wide range of cognitive and emotional disorders. Moreover, nearly all previous studies have used convenience samples of patients referred to clinical care for their motor symptoms.

In the most comprehensive study to date, Brown and MacCarthy ${ }^{4}$ used a standardised psychiatric interview designed to assess psychiatric disturbances in the general population to provide a detailed description of the psychiatric symptomatology in 40 patients with Parkinson's disease. Seventy per cent of the patients exhibited at least one psychiatric syndrome, the most common conditions were depression and anxiety. However, only $10 \%$ of the patients warranted a psychiatric diagnosis, and these were all within the class "neurotic disorders". The authors acknowledged that "analysis on the level of diagnostic category is probably unsuitable when considering patients with Parkinson's disease". Furthermore, the finding that 24 of the 37 psychiatric syndromes were not represented in the sample suggests that instruments aimed at identifying general psychiatric syndromes are not well suited for a population with a brain disorder such as Parkinson's disease.

In the current study, we used an instrument specifically designed to assess psychopathology in patients with brain disorders, the neuropsychiatric inventory $(\mathrm{NPI})^{5}$ to investigate the range of neuropsychiatric syndromes in a sample of patients with Parkinson's disease. The NPI has proved validity and reliability in patients with dementia. It is a convenient instrument that assesses both the frequency and severity of 10 syndromes: delusions, hallucinations, agitation, depression, anxiety, euphoria, apathy, disinhibition, irritability, and abnormal motor output. The patient population assessed was demographically representative and was well characterised clinically and cognitively.

\section{Patients and methods}

PATIENTS

Patients were recruited from an epidemiological study of Parkinson's disease in Rogaland county, Norway. The study area has 220000 inhabitants. Total ascertainment of patients with recognised idiopathic Parkinson's disease in this defined geographical area was attempted. The 
main source of patient information was the files of the outpatient clinic of the Department of Neurology at the Central Hospital of Rogaland, that serves the entire area in a public health system. Additional sources were the main patient files of the hospital. These files included information on diagnosis of every inpatient referred to the hospital since 1972. Furthermore, all general practitioners, nursing homes, consulting physicians in charge of nursing homes, district nurses, and home health care workers in the study area were contacted in writing and asked for information on patients with symptoms of parkinsonism. Information on all members of the local branch of the Parkinson's disease society was available as well. All nursing home physicians, district nurses and home health workers, and $75 \%$ of the general practitioners responded to the written inquiry. Based on these sources, nearly 500 patients were considered for a diagnostic evaluation. Based on information from the hospital records, some patients could be excluded because they did not have Parkinson's disease. Nearly 400 patients were interviewed and examined by one of the neurologists in the study group, and 245 were diagnosed as having Parkinson's disease. The baseline evaluation was carried out in 1992 and 1993. ${ }^{6}$ During 1996 and 1997, the survivors were invited to participate in the present study and assessed with a comprehensive evaluation consisting of three sessions: a neurological, psychiatric, and neuropsychological examination. The three evaluations were performed within 1 month of each other. To evaluate the representativeness of this follow up sample, comparisons with the baseline population were performed. The patients provided informed consent, and the study was approved by the regional ethics committee, University of Bergen.

\section{METHODS}

Diagnosis and clinical evaluation of Parkinson's disease

A semistructured interview was performed to obtain information on disease history, drug therapy, response to levodopa, and demographic variables. The clinical examination of motor symptoms consisted of a complete unified Parkinson's disease rating scale (UPDRS) assessment ${ }^{7}$ including the Hoehn and Yahr scale. ${ }^{8}$ The diagnostic evaluation was based on clinical information at the primary evaluation, disease development, and response to levodopa. To achieve high diagnostic accuracy without losing sensitivity, a diagnostic classification defining clinical definite, probable, and possible Parkinson's disease was used (see Larsen et $a l^{\dagger}$ for a description of the categories). The same assessment had been conducted during the evaluation 4 years previously. Other neurological diagnoses or use of neuroleptic drugs before the first diagnostic evaluation prohibited a diagnosis of Parkinson's disease. In 40 patients CT or MRI of the brain was performed, without abnormalities compatible with diagnosis other than Parkinson's disease. Patients with clinical fluctuations were examined in the "on state".
CLINICAL SUBGROUPS

The UPDRS motor scale was used to obtain scores for several clinical factors that might have an impact on psychiatric symptoms. We calculated a tremor score by adding scores on item 20 and 21 (tremor at rest and action or postural tremor of hands). Rigidity was measured by the scores on item 22, and akinesia by adding the scores on items 23-26 (finger taps, hand movements, rapid alternating movements of hand, and leg agility). We calculated right and left side motor subscores by adding the respective scores for right and left side on UPDRS items 20-26, and identified patients with right or left dominance by using a cut off score of \pm 3 or more on the difference between scores for the right and left sides.

\section{Cognitive assessment}

A semistructured interview based on the diagnostic and statistical manual of mental disorders, third edition, revised ${ }^{10}$ (DSM-III-R) criteria for dementia was administered to the caregiver by a psychiatrist with experience in geriatric psychiatry and neuropsychiatry (NGL and DA). Two brief cognitive screening instruments were administered to the patient by a research nurse: the mini mental state examination (MMSE) $)^{11}$ and the dementia rating scale (DRS). ${ }^{12}$ The DRS provides a total score and five subscores: attention, memory, categorisation, initiation and perseveration, and construction. Patients with an MMSE score of 16 or above performed a neuropsychological battery assessing the following cognitive domains: executive functioning (Stroop test), visual memory (Benton visual retention test), visuospatial functioning (judgement of line orientation test) and attention and vigilance. A group of healthy elderly controls $(n=37)$ performed the cognitive screening and neuropsychological evaluation. The final diagnosis of dementia (yes, no, questionable) according to the DSM-III-R criteria was made by two of us (DA and JPL), based on the clinical interview, the cognitive screening, and the neuropsychological tests.

\section{Neuropsychiatric assessment}

The 10 item version of the NPI was administered by one of the two psychiatrists (NGL and DA). For patients living in their homes, the NPI was administered to a family member familiar with the patient's behaviour, usually the spouse. For patients living in nursing homes, the nurse or another staff member who knew the patient best was interviewed.

The NPI was administered following the procedures previously described. ${ }^{5}$ Screening questions for each of the 10 neuropsychiatric symptoms were posed first. If a positive response was obtained, then the symptom was explored with scripted questions that focused on specific features of the neuropsychiatric symptom. The caregiver then rated the symptom; scores from 1 through 4 were obtained for the frequency and 1 through 3 for the severity of each symptom. A composite score for each symptom was the product of the frequency and 
Mean subscores of neuropsychiatric inventory items in the total sample and among patients with the symptom present

\begin{tabular}{|c|c|c|c|c|c|c|c|}
\hline & \multicolumn{2}{|c|}{$\begin{array}{l}\text { Total sample } \\
(n=139)\end{array}$} & \multicolumn{5}{|c|}{$\begin{array}{l}\text { Patients with symptom present } \\
\text { (non-zero score) }\end{array}$} \\
\hline & Mean & $S D$ & $n$ & $\begin{array}{l}\% \text { Of } \\
\text { sample }\end{array}$ & $\begin{array}{l}\text { Mean } \\
\text { score }\end{array}$ & $S D$ & Range \\
\hline Delusions & 0.5 & 1.6 & 22 & 15.8 & 3.3 & 2.6 & $1-12$ \\
\hline Hallucinations & 0.9 & 2.2 & 37 & 26.6 & 3.6 & 2.9 & $1-12$ \\
\hline Agitation & 0.7 & 2.1 & 23 & 16.5 & 4.3 & 3.5 & $1-12$ \\
\hline Depression & 1.5 & 2.6 & 53 & 38.1 & 3.9 & 2.9 & $1-12$ \\
\hline Anxiety & 1.0 & 2.7 & 28 & 20.1 & 5.1 & 4.0 & $1-12$ \\
\hline Euphoria & 0.01 & 0.2 & 1 & 0.7 & & & \\
\hline Apathy & 1.1 & 2.9 & 23 & 16.5 & 6.7 & 3.7 & $1-12$ \\
\hline Disinhibition & 0.2 & 0.9 & 9 & 6.5 & 2.7 & 2.3 & $1-8$ \\
\hline Irritability & 0.4 & 1.9 & 14 & 10.1 & 4.4 & 4.2 & $1-12$ \\
\hline Aberrant motor behaviour & 0.7 & 2.3 & 15 & 10.8 & 6.1 & 3.9 & $1-12$ \\
\hline Neuropsychiatric inventory total & 7.1 & 12.0 & 85 & 61.2 & 11.6 & 13.5 & $1-72$ \\
\hline
\end{tabular}

severity subscores (maximum=12). The composite score was used in the analysis.

\section{STATISTICS}

Analyses consisted primarily of description of NPI scores and calculation of proportions. The relations of NPI scores to demographic and clinical variables were analysed using nonparametric tests (Spearman rank correlation and Mann-Whitney $U$ test). All $\mathrm{p}$ values are two tailed. A significance level of 0.01 was chosen due to a large number of comparisons.

\section{Results}

CLINICAL AND DEMOGRAPHIC CHARACTERISTICS

Of the 245 patients evaluated in 1993, 88 had died, and seven subsequently had been diagnosed as not having Parkinson's disease. Accordingly, 150 patients were invited to participate in the present study. Eleven subjects refused to participate, and hence a total of 139 patients $(92.7 \%$ of the survivors with Parkinson's disease) completed the follow up study. The male:female ratio was $61: 78$, the mean age was 74.4 (SD 7.9) years, and the duration of Parkinson's disease was 12.6 (SD 5.1) years. Thirty six patients $(26 \%)$ lived in a nursing home. Most were diagnosed as clinical definite Parkinson's disease $(n=104,74.8 \%$ of the sample), 32 (23\%) as probable Parkinson's disease, and three $(2.2 \%)$ as possible Parkinson's disease. Ten patients $(7.2 \%)$ were in Hoehn and Yahr stage 1 or $1.5,40(28.8 \%)$ in stage 2 or $2.5,34(24.5 \%)$ in stage $3,32(23 \%)$ in stage 4 , and $21(15.1 \%)$ in stage 5 . (Data were missing for two patients). Fifty patients, $36 \%$ of the sample, met the DSM-III-R criteria for dementia, and eight had questionable dementia. The patients with dementia were significantly older than the non-demented patients (78.6 (SD 5.9) $(\mathrm{p}<0.001))$. An MMSE score was obtained for 127 of the patients, and the mean score was 25.2 (SD 5.9). Nine of the 12 who did not complete the MMSE were too demented to perform the test. One hundred and twelve completed the DRS; the mean score was 127.5 (SD 22.4). Eighteen of the 27 noncompleters were too demented to perform the test. The demographic and clinical characteristics of the study sample were similar to those in the population of patients with Parkinson's disease from the evaluation in 1993, with the expected changes in conjunction with the evolution of more advanced disease. ${ }^{6}$
NEUROPSYCHIATRIC INVENTORY SCORES

Interrater reliability of the NPI was assessed by the two raters performing simultaneous ratings of 12 patients. Intraclass correlations between the two raters on the NPI items were high: hallucinations (0.94), depression (0.84), anxiety (0.94), apathy (0.84), aberrant motor behaviour (0.96), and total NPI score (0.93). All 10 symptoms were reported in the sample, but for the other items, too few symptoms were detected and thus the reliability data could not be calculated. Fifty four patients with Parkinson's disease (39\%) had no scorable symptoms on the NPI. Twenty two patients (16\%) had one symptom, 28 (20\%) had two, and 35 $(25 \%)$ had three or more neuropsychiatric symptoms present. The most common behaviours were depression (present in 38\% of the patients), hallucinations (27\%), and anxiety $(20 \%)$. The least common symptoms were euphoria $(0.7 \%)$ and disinhibition $(6.5 \%)$. The highest mean scores were found for depression, apathy, and hallucinations. When severity scores were calculated only for those patients in whom the symptom was present, the highest scores were obtained for apathy, aberrant motor behaviour, and anxiety. The scores and frequency of all symptoms are shown in the table.

RELATIONS AMONG NEUROPSYCHIATRIC

INVENTORY SUBSCORES

High correlations were found among delusions, hallucinations, agitation, and irritability; and between apathy and anxiety. Depression correlated with both the psychotic and agitation symptoms and anxiety and apathy symptoms. A principal component factor analysis was performed to further explore the interrelation of the NPI symptoms. Consistent with the correlation analysis, two factors emerged with an eigenvalue of at least 1.5: factor 1 included the items delusion, hallucination, and irritability, and factor 2 included anxiety and apathy.

The relation between apathy and depression was explored in more detail. Six of the 23 patients with apathy ( $4.3 \%$ of the sample) had no evidence of depression (score of 0 ), whereas the remaining patients with apathy had positive scores on the depression subscale.

RELATION BETWEEN NPI SCORES AND CLINICAL AND DEMOGRAPHIC VARIABLES

The clinical and demographic correlates of neuropsychiatric symptoms in Parkinson's disease were explored. Neither age or duration of disease nor levodopa dosage correlated with the NPI subscores. A relation between NPI scores and Hoehn and Yahr stage was found. Most behaviour scores increased with more advanced disease, and significant correlations with the Hoehn and Yahr stage were found for total NPI score (Spearman's $r=0.37, \mathrm{p}<0.001$ ), and delusion $(r=0.38, \mathrm{p}<0.001)$, hallucination $(r=0.37, \mathrm{p}<0.001)$, agitation $(r=0.28$, $\mathrm{p}<0.001)$, and aberrant motor behaviour $(r=0.28, \mathrm{p}<0.001)$ scores. Patients in nursing homes had higher total NPI scores than home dwelling patients $(U=1077, \mathrm{p}<0.001)$, and this difference was primarily due to higher scores 
on the delusion, hallucination, agitation, and disinhibition subscales. Eighty three per cent of the nursing home population had at least one symptom present. Analysis of the relation between neuropsychiatric syndromes and sex showed somewhat more irritability among male patients $(U=2033, \mathrm{p}<0.005)$.

Scores of delusions, hallucinations, and agitation correlated significantly with the akinesia and rigidity scores $(\mathrm{p}<0.005)$. No other significant correlations were found between NPI subscores and scores of akinesia, rigidity, or tremor. Subscores for NPI did not differ between patients with predominantly right $(n=26)$ or left sided $(n=38)$ parkinsonism, but a non-significant trend towards a higher total NPI score in the left side group was found $(U=373 ; \mathrm{p}=0.087)$. Patients with probable and possible Parkinson's disease showed a trend towards higher NPI total score $(U=1375$; $\mathrm{p}=0.03)$ and agitation score $(U=1480, \mathrm{p}=0.02)$ compared with patients with clinical definite Parkinson's disease. There were no significant correlations between NPI scores and duration and level of disability caused by dyskinesias. "On-off" fluctuations were reported in $45 \%$ of the patients, but no relations between NPI subscores and fluctuations were found.

RELATION BETWEEN NPI SCORES AND COGNITIVE FUNCTIONS

A characteristic relation was found between NPI subscores and cognitive functions. Demented patients had more frequent and more severe neuropsychiatric morbidity as measured with the NPI total score compared with patients without dementia or with questionable dementia $(U=1106, \mathrm{p}<0.001)$. Significant relations with dementia were found for delusions $(U=1545, \mathrm{p}<0.001)$, hallucinations $(U=1228$, $\mathrm{p}<0.001)$, apathy $(U=1570, \mathrm{p}<0.05)$, and aberrant motor behaviour $(U=1638, \mathrm{p}<0.001)$. The relations between the NPI items and tests measuring different cognitive functions were also explored. A distinct pattern of relations emerged. Total NPI, hallucination, delusion, and agitation scores correlated with MMSE, most DRS subscales, and performance on memory tests. In addition, a significant correlation was found between apathy and the number of errors on the Stroop test, a measure of executive functioning.

\section{Discussion}

The objective of this study was to explore the range and profile of the psychiatric morbidity in Parkinson's disease, and to examine the relation between psychiatric symptoms and demographic and clinical variables. The main finding was that $61 \%$ of the sample showed at least one psychiatric symptom, and $45 \%$ had two or more. The proportion was particularly high among the Parkinson's disease population living in nursing homes. Depression was the most common symptom, followed by hallucinations and anxiety. The highest scores were reported for apathy, anxiety, and depression. The rates found were similar to those reported in previous studies, focusing on depression, ${ }^{1}$ hallucinations, ${ }^{2}$ anxiety, ${ }^{13}$ and apathy ${ }^{14}$ and are well above the frequencies found in the general, non-parkinsonian elderly population in Scandinavia ${ }^{15} 16$

The severity of psychiatric symptoms were associated with disease stage and cognitive impairment, but not with age, duration of disease, levodopa dose, dyskinesia, or on-off phenomena. These findings are in accordance with those reported by Brown and MacCarthy. Depression did not correlate with clinical or cognitive variables although it was more often present in those with other neuropsychiatric symptoms. The lack of association between depression and disease stage is in accordance with previous studies, ${ }^{11718}$ and suggests that the mood changes in Parkinson's disease are not a reaction to motor disability but rather reflect a neurobiologically based disorder of emotional functions. The previously reported relation between depression and akinetic-rigid subtype $^{17}$ and right sided Parkinson's disease ${ }^{19}$ were not replicated in this study.

To our knowledge, only one previous study has investigated the range of psychiatric morbidity in Parkinson's disease. ${ }^{4}$ Compared with the general population of patients with Parkinson's disease ${ }^{6}$ the sample in that study was a highly selected convenience group, with a lower age and few men. Furthermore, they were cognitively intact and had a more restricted range of severity of disease, two factors which probably influenced the range and severity of psychiatric morbidity. Our sample was more representative, but included only patients with a disease duration of at least 4 years. However, as both studies showed, duration of disease did not seem to influence psychiatric morbidity. Different methods for assessing psychiatric symptoms were used in the two studies. In the current study, the NPI was used, an instrument designed to identify psychiatric morbidity in patients with neurological disorders. ${ }^{5}$ Nevertheless, the findings in these two studies regarding the rate of psychiatric morbidity in general are similar, and show a high prevalence of psychiatric morbidity in patients with Parkinson's disease.

The study has methodological shortcomings which need to be recognised when interpreting the findings. Because we did not include a control group, the exact prevalence of psychiatric symptoms specific to Parkinson's disease cannot be estimated with certainty. However, other studies suggest that patients without brain disease have very low frequencies of NPI symptoms present,${ }^{5}$ indicating that most of the psychiatric morbidity is in fact caused by Parkinson's disease. The sample is drawn from the survivors of a population of patients with Parkinson's disease, but the sample is skewed towards patients with a long duration of disease and thus with more advanced illness. This might have increased the prevalence of psychiatric symptoms. The strengths of the study are the relatively large sample, including institutionalised and severely ill patients, and the comprehensive evaluation battery including a standardised assessment of neuropsychiatric symptoms with high validity and reliability. 
The psychiatric symptoms clustered into two groups, which had different relations with the cognitive and motor domains of the disease. One group of symptoms included delusions, hallucinations, agitation, and irritability. These symptoms correlated with levels of akinesia and rigidity, and with impairment on general cognitive measures such as the MMSE score and memory. Another cluster of symptoms were apathy and anxiety. Apathy usually occurred together with depression, consistent with other studies. ${ }^{14}{ }^{20}$ This is different from the findings in other neurobehavioural disorders such as progressive supranuclear palsy and Alzheimer's disease, in which apathy is less associated with depression. ${ }^{20}$ Apathy was also associated with impairment of executive functions. The pattern of relation between emotional and cognitive abnormalities in Parkinson's disease suggests that psychosis is related to abnormalities of temporolimbic structures mediating mnemonic functions, whereas disturbances of frontal systems, also mediating executive skills, might account for the occurrence of apathy. Psychosis was more common in patients meeting criteria for dementia than in those with preserved cognition. In the initial phase of this study, we found that patients with Parkinson's disease with dementia were more likely to exhibit psychotic symptoms, ${ }^{21}$ similar to other investigations. ${ }^{22}$ This may reflect the presence of cortical Lewy bodies in the paralimbic cortex or more marked cholinergic deficits found in these patients.

In conclusion, the present study shows a high prevalence of psychiatric morbidity among patients with Parkinson's disease. The rate of reported neuropsychiatric features is comparable with that reported in other brain diseases, such as progressive supranuclear palsy, ${ }^{23} \mathrm{Hunt}-$ ington's disease, ${ }^{24}$ frontotemporal dementia, ${ }^{25}$ and Alzheimer's disease. ${ }^{26}$ However, the pattern of psychiatric symptoms differs among different brain disorders, reflecting the involvement of different brain regions and different transmitter systems. Clinicopathological or imaging studies are needed to clarify these relations in more detail. This study emphasises the importance of psychiatric symptoms in Parkinson's disease, which were present in most patients. Clinicians should focus on the emotional and cognitive disturbances as well as the motor manifestations of the disease.

This project was supported by the Research Councils of the Central and Psychiatric Hospitals of Rogaland, a Scandinavian
Society of Psychopharmacology grant, NIA Alzheimer's Disease Center grant AG10123, an Alzheimer's Disease Center of California grant, and the Sidell-Kagin Foundation.

1 Cummings JL. Depression and Parkinson's disease: a review. Am F Psychiatry 1992;149:443-54.

2 Cummings JL. Behavioral complications of drug treatment of Parkinson's disease. F Am Geriatr Soc 1991;33:708-16.

3 Dubois B, Pillon B. Cognitive deficits in Parkinsons disease. 7 Neurol $1997 ; 244: 2-8$

4 Brown RG, MacCarthy B. Psychiatric morbidity in patients with Parkinson's disease. Psychol Med 1990;20:77-87.

5 Cummings JL, Mega M, Gray K, et al. The neuropsychiatric inventory: comprehensive assessment of psychopatholric inventory: comprehensive assessment of psyct

6 Tandberg E, Larsen JP, Nessler EG, et al. The epidemiology of Parkinson' disease in the county of Rogaland, Norway. Mov Disord 1995;10:541-9.

7 Fahn S, Elton RL. Members of the UPDRS Development Committee. Unified Parkinson's disease rating scale. In: Fahn S, Marsden CD, Calne DB, et al, eds. Recent development in Parkinson's disease. Florham Park, NJ: Macmillan Health Care Information, 1987:153-63.

8 Hoehn MH, Yahr MD. Parkinsonism: onset, progression, and mortality. Neurology 1967;17:427-42.

9 Larsen JP, Dupont E, Tandberg E. The clinical diagnosis of Parkinson's disease: proposal of diagnostic subgroups classified at different levels of confidence. Acta Neurol Scand 1994;84:242-51.

10 Diagnostic and Statistical Manual of Mental Disorders, 3rd ed, revised. Washington, DC: American Psychiatric Association, 1987.

11 Folstein MF, Folstein SE, McHugh PR. Mini-mental state: a practical method for grading the mental state of patients for the clinician. F Psychiatr Res 1975;12:189-98.

12 Mattis S. Dementia rating scale. In: Bellak L, Karasu TB, eds. Geriatric psychiatry. A handbook for psychiatrists and primary care physicians. ew York: Grune and Stratton, 1976:108-121.

13 Richard IH, Schiffer RB, Kurlan R. Anxiety and Parkinson's disease. F Neuropsychiatry Clin Neurosci 1996;8:383-92.

14 Starkstein SE, Mayberg HS, Preziosi TJ, et al. Reliability, validity and clinical correlates of apathy in Parkinson's disvalidity and clinical correlates of apathy in Parkinson's

15 Skoog I, Nilsson L, Landahl S, et al. Mental disorders and the use of psychotropic drugs in an 85-year-old urban population. Int Psychogeriatr 1993;5:33-48.

16 Forsell Y, Winblad B. Feelings of anxiety and associated variables in a very elderly population. International fournal of Geriatric Psychiatry 1998;13:454-8.

17 Starkstein SE, Petracca G, Chemirinski E, et al. Depression in classic versus akinetic-rigid Parkinson's disease. Mov in classic versus akinetic

18 Tandberg E, Larsen JP, Aarsland D, et al. Risk factors for depression in Parkinson's disease. Arch Neurol 1997;54: 625-30.

19 Cole SA, Woodard JL, Juncos JL, et al. Depression and disability in Parkinson's disease. $\mathcal{F}$ Neuropsychiatry 1996;8:205.

20 Levy M, Cummings JL, Fairbansk LA, et al. Apathy is not depression. I Neuropsychiatry Clin Neurosci 1998;10:314-9.

21 Aarsland D, Larsen JP, Cummings JL. Prevalence and clinical correlates of psychosis in Parkinson's disease. Arch Neucal correlates of psychosis
rol 1999;56:595-601.

22 Sanchez-Ramos JR, Ortoll R, Paulson GW.Visual hallucinations in Parkinson's disease. Arch Neurol 1996;53:1263-8.

23 Litvan I, Mega MS, Cummings JL, et al. Neuropsychiatric aspects of progressive supranuclear palsy. Neurology 1996; 47:1184-9.

24 Litvan I, Paulsen JS, Mega MS, et al. Neuropsychiatric assssment of patients with hyperkinetic and hypokinetic movement disorders. Arch Neurol 1998;55:1313-19.

25 Levy ML, Miller BL, Cummings JL, et al. Alzheimer disease and frontotemporal dementias. Behavioural distinctions. and frontotemporal dementias.

Arch Neurol 1996;53:687-90.
26 Mega M, Cummings JL, Fiorello T, et al. The spectrum of behavioral changes in Alzheimer's disease. Neurology 1996; 46:130-5. 